\title{
The speed interval: a rotation algorithm for endomorphisms of the circle
}

\author{
JAN BARKMEIJER \\ Mathematisch Instituut, Postbus 800, 9700 AV Groningen, The Netherlands \\ (Received 12 January 1987)
}

Abstract. Let $f$ be a continuous map of the circle into itself of degree one. We introduce the notion of rotation algor thms. One of these algorithms associates each $z \in S^{1}$ with an interval, the so-called speed interval $S(z, f)$, which is contained in the rotation interval $\rho(f)$ of $f$. In contrast with the rotation set $\rho(z, f)$, the interval $S(z, f)$ sometimes allows us to ascertain that $\rho(f)$ is non-degenerate, by using only finitely many elements of $\left\{f^{n}(z) \mid n \geq 0\right\}$. We further show that all choices for $\rho(z, f)$ and $S(z, f)$ occur, for certain $z \in S^{1}$, provided that $\rho(z, f) \subset S(z, f) \subset \rho(f)$.

\section{Introduction and statement of results}

Let $\operatorname{End}_{1}^{0}\left(S^{1}\right)$ be the set of continuous endomorphisms of degree one of the circle. For an $f \in \operatorname{End}_{1}^{0}\left(S^{1}\right)$ a lift $F$ is a continuous map on $\mathbb{R}$ such that $f \circ \pi=\pi \circ F$, where $\pi: \mathbb{R} \rightarrow S^{1}$ is the natural projection, $\pi(t)=e^{2 \pi i t}$. So a lift is determined uniquely up to shifts by integers. Since $f$ is of degree one we have

$$
F(x+1)=F(x)+1 \quad \text { for all } x \in \mathbb{R} .
$$

If $f$ is monotone, i.e. $x<y$ implies $F(x) \leq F(y)$, then the limit of $\left(F^{n}(x)-x\right) / n$ for $n \rightarrow \infty$ exists for all $x \in \mathbb{R}$ and it is independent of $x$, cf. [10]. So to a monotone map $f$ we can assign a number, unique modulo one, the so-called rotation number $\rho(f)$ of $f$.

In the general case of endomorphisms the above limit may not exist and if it exists it may be dependent on $x$. For that reason the concept of the rotation set was introduced, cf. [9].

The rotation set $\rho(z, f)$ of $z$ under $f$ is given by

$$
\rho(z, f)=\left\{\text { limit points of }\left\{\frac{F^{n}(x)-x}{n}\right\}_{n \geq 1} \text { with } \pi(x)=z\right\} .
$$

and the rotation interval $\rho(f)$ of $f$ is defined by

$$
\rho(f)=\left\{\text { limit points of }\left\{\frac{F^{n}(x)-x}{n}\right\}_{n \geq 1} \mid x \in \mathbb{R}\right\} .
$$

Note that these sets are again uniquely determined up to shifts by integers. The choice for $x$ from $\pi^{-1}(z)$ does not influence the rotation set $\rho(z, f)$. Both sets form an interval and in [7] it is shown that each $\alpha \in \rho(f)$ is realized as rotation number 
of some point $z \in S^{1}$ :

$$
\lim _{n \rightarrow \infty} \frac{F^{n}(x)-x}{n}=\alpha, \quad \pi(x)=z .
$$

An endomorphisms with non-degenerate rotation interval also has various periodic points. A period point $z$ of $f$ is a point such that $f^{n}(z)=z$, for some $n \geq 1$. The least integer for which this is the case is called the period of $z$. Because $f^{n}(z)=z$, there exists an integer $m$ such that $F^{n}(x)=x+m, \pi(x)=z$. Then $\rho(z, f)=p / q$ is the rotation number of $z$, where $p / q=m / n$ and $(p, q)=1$. The set $\left\{f^{i}(z) \mid i=0, \ldots, n-1\right\}$ is called a periodic orbit of $f$ with rotation number $p / q$ and period $n$.

THEOREM A (see [8]). Let $f \in \operatorname{End}_{1}^{0}\left(S^{1}\right)$. If $k / n \in$ int $\rho(f)$ then $f$ has a periodic point of period $n$.

Given a rotation interval $\rho(f)$ the set $P(f)=\{n \mid f$ has a periodic point of period $n\{$ is determined in [8], [2]. Further [4] gives a complete description of all rotation sets $\rho(z, f)$ in terms of the rotation interval $\rho(f)$ :

THEOREM B. If $f \in \operatorname{End}_{1}^{0}\left(S^{1}\right)$ then:

(i) $\rho(z, f)$ is a closed subinterval of $\rho(f)$ for all $z \in S^{1}$;

(ii) given $[\alpha, \beta] \subset \rho(f), \alpha \leq \beta$, there exists $z \in S^{1}$ such that $\rho(z, f)=[\alpha, \beta]$.

So in contrast with monotone maps where each point on $S^{1}$ has the same asymptotic progression, it may occur in the general case that the asymptotic progression of a point never settles down to a limit value. Some difficulties arise in deciding whether a map $f$ exhibits this dynamics or equivalently in determining whether $\rho(f)$ is non-degenerate. For if one starts computing $\rho(z, f)$ the complete orbit orb $(x, F)=$ $\left\{F^{n}(x) \mid n=0,1, \ldots\right\}$, where $x \in \pi^{-1}(z)$, is needed.

Yet we want to analyse the dynamics of $f$ by studying the progression in orb $(x, F)$. It will appear that although a finite part of orb $(x, F)$ does not say anything about $\rho(z, f)$ it may give information about $\rho(f)$.

In $\S 2$ we present some algorithms which sometimes make it possible to conclude that $\rho(f)$ is non-degenerate with only a finite part of orb $(x, F)$ for some $x \in \mathbb{R}$. More precisely, if for a finite part of an orbit such an algorithm yields an interval $I$ then $I \subset \rho(f)$. One of the algorithms we give, associates each $z \in S^{1}$ with an interval $S(z, f) \subset \rho(f)$, the speed interval of $z$ under $f$. The interval $S(z, f)$ is a topological invariant: if $h: S^{1} \rightarrow S^{1}$ is an orientation preserving homeomorphism, then

$$
S(z, f)=S\left(h(z), h f h^{-1}\right) .
$$

The main result we shall prove is that $\rho(z, f)$ and $S(z, f)$ are in a sense independent:

THEOREM. Let $f \in \operatorname{End}_{1}^{0}\left(S^{1}\right)$. For every two closed intervals I and $J$, possibly degenerate, such that $I \subset J \subset \rho(f)$ there exists $z \in S^{1}$ with

(i) $\rho(z, f)=I$ and

(ii) $S(z, f)=J$.

In $\S \S 3$ and 4 we derive some ingredients essential in proving the theorem. Finally the proof of the theorem is given in $\S 5$. 
Acknowledgement. I wish to express my thanks to Floris Takens, for his comments and for the stimulating discussions we had preparing the manuscript.

\section{Rotation algorithms}

Before we give an example of a rotation algorithm we first explain the concept of such an algorithm.

A rotation algorithm $A$ is defined in terms of two other algorithms, which we denote by $A^{-}$and $A^{+}$in the sequel. Both these algorithms $A^{-}$and $A^{+}$assign to an orbit interval a real number.

An orbit interval $X$ of $F$ is a set of points of the form $\left\{F^{i}(a)\right\}_{i=0}^{n}$ with $F$ a lift of $f \in \operatorname{End}_{1}^{0}\left(S^{1}\right)$ and $0 \leq n \leq+\infty$. If $X=\left\{x_{i}\right\}_{i=0}^{m}$ and $Y=\left\{y_{j}\right\}_{j=0}^{n}$ are orbit intervals we say $X$ is included in $Y$, in notation $X \sqsubset Y$, if and only if there exist natural numbers $n_{0}$ and $k$ such that

$$
x_{i}=y_{n_{0}+i}+k \quad \text { for } i=0, \ldots, m .
$$

Intuitively one may think of $A^{-}(X)$ or $A^{+}(X), X$ an orbit interval of $F$, as a kind of approximation of $\rho^{-}(f)$ or $\rho^{+}(f)$ respectively, where $\rho(f)=\left[\rho^{-}(f), \rho^{+}(f)\right]$. We remark that we do not necessarily have $A^{-}(X) \leq A^{+}(X)$.

Next we state some properties which $A^{-}$and $A^{+}$at least should satisfy if they are related to a rotation algorithm.

(R1) $\rho^{-}(f) \leq A^{-}(X)$ and $A^{+}(X) \leq \rho^{+}(f)$ for all orbit intervals $X$ of $F$.

(R2) If $X$ and $Y$ are orbit intervals of $F$ and $X \sqsubset Y$ then:

$$
A^{-}(Y) \leq A^{-}(X) \text { and } A^{+}(X) \leq A^{+}(Y)
$$

(R3) $A^{-}($orb $(x, F)) \leq A^{+}($orb $(x, F))$ for all $x \in \mathbb{R}$.

To each $z \in S^{1}$ we assign an interval $A(z, f)$ by putting

$$
A(z, f)=\left[A^{-}(\operatorname{orb}(x, F)), A^{+}(\text {orb }(x, F))\right] \quad \text { where } \pi(x)=z .
$$

Some remarks. (a) The interval $A(z, f)$ is well defined. In principle it may depend on the choice of the lift $F$ of $f$. For the algorithms we shall consider, $A(z, f)$ is uniquely determined up to translation by integers.

(b) The interval $A(z, f)$ is finitely determined: if for a finite orbit interval $Y \sqsubset \operatorname{orb}(x, F), \pi(x)=z$, we have $\left[A^{-}(Y), A^{+}(Y)\right]=I$ with $I$ non-degenerate then $I \subset A(z, f)$ and further $I \subset \rho(f)$.

(c) The choice of $x$ from $\pi^{-1}(z)$ does not influence $A(z, f)$.

The rotation set is constant on orb $(z, f)=\left\{f^{n}(z) \mid n \geq 0\right\}$, i.e. $\rho(z, f)=\rho\left(f^{n}(z), f\right)$ for all $n \geq 0$. For the interval $\boldsymbol{A}(z, f)$ we have:

LEMMA 2.1. Let $f \in \operatorname{End}_{1}^{0}\left(S^{1}\right)$. If $z$ is a periodic point of $f$ then

$$
A(w, f)=A(z, f) \quad \text { for all } w \in \text { orb }(z, f) \text {. }
$$

Proof. Choose $x \in \pi^{-1}(w)$ and $y \in \pi^{-1}(z)$. Write $X=$ orb $(x, F)$ and $Y=\operatorname{orb}(y, F)$ then $X \sqsubset Y$ and $Y \sqsubset X$. By property $(\mathrm{R} 2)$ this gives $A^{-}(X)=A^{-}(Y)$ and $A^{+}(X)=$ $A^{+}(Y)$, consequently $A(w, f)=A(z, f)$. 
We shall look at so-called two point rotation algorithms. By this we mean a rotation algorithm which is based on the information about $\rho(f)$ obtained from pairs of elements of an orbit interval. The definition of the rotation set $\rho(z, f)$ motivates such a two point algorithm. For convenience we recall it here:

$$
\rho(z, f)=\left[\liminf _{n \rightarrow \infty} \frac{F^{n}(x)-x}{n}, \limsup _{n \rightarrow \infty} \frac{F^{n}(x)-x}{n}\right] .
$$

Let $Y=\left\{F^{n}(x)\right\}_{n=0}^{N}, 0 \leq N \leq+\infty$, be an orbit interval. Define $M_{\alpha \alpha}^{-}$and $M_{\alpha}^{+}, \alpha \in \mathbb{R}$, by

and

$$
M_{\alpha}^{-}(Y)=\inf _{N \geq j>i \geq 0} \frac{F^{j}(x)-F^{i}(x)+\alpha}{j-i}
$$

$$
M_{\alpha}^{+}(Y)=\sup _{N \geq j>i \geq 0} \frac{F^{j}(x)-F^{i}(x)-\alpha}{j-i} .
$$

We write $M_{\alpha}^{-}(z, f)=M_{\alpha}^{-}($orb $(x, F))$ and $M_{\alpha}^{+}(z, f)=M_{\alpha}^{+}($orb $(x, F))$ with $\pi(x)=z$. In this way $M_{\alpha}^{-}$and $M_{\alpha}^{+}$define maps on $S^{1}$ which satisfy a semi-continuity property.

A map $g: S^{1} \rightarrow \mathbb{R}$ is called lower- or upper semi continuous if for each $t \in \mathbb{R}$, the set $\left\{z \in S^{1} \mid g(z)>t\right\}$ or $\left\{z \in S^{1} \mid g(z)<t\right\}$ respectively is open in $S^{1}$.

LEMMA 2.2. Let $f \in \operatorname{End}_{1}^{0}\left(S^{1}\right)$ and $\alpha, t \in \mathbb{R}$.

(i) The maps $M_{\alpha}^{-}$and $M_{\alpha}^{+}$are upper- and lower semi-continuous respectively;

(ii) if $M_{\alpha}^{-}(z, \tilde{f})<t$ or $M_{\alpha}^{+}(z, \tilde{f})>t$ then there is a $C^{0}$-neighbourhood. $V$ of $\tilde{f}$ such that $M_{\alpha}^{-}(z, f)<t$ or $M_{\alpha}^{+}(z, f)>t$ respectively, for $f \in V$.

Proof. (i) It suffices to show the upper semi continuity of $M_{\alpha}^{-}$; the other case is similar. Fix $\alpha \in \mathbb{R}$, we prove that $U_{t}=\left\{z \in S^{1} \mid M_{\alpha}^{-}(z, f)<t\right\}, t \in \mathbb{R}$, is open in $S^{1}$. We may assume that $U_{t} \neq \varnothing$. For $w \in U_{t}$ we have, by taking $N$ sufficiently large,

$$
\inf _{N \geq j>i \geq 0} \frac{F^{j}(x)-F^{i}(x)+\alpha}{j-i}<t \quad \text { with } x \in \pi^{-1}(w)
$$

Because of the continuity of $F$ there exists a neighbourhood $W$ of $x$ such that

$$
\inf _{N \geq j>i \geq 0} \frac{F^{j}(y)-F^{i}(y)+\alpha}{j-i}<t \quad \text { for } y \in W .
$$

Hence $M_{\alpha}^{-}(z, f)<t$ for all $z \in \pi(W)$. We conclude that $U_{t}$ is open in $S^{1}$. Statement (ii) is similar to (i), we omit the analogous proof.

Since $M_{r}^{-}(z, f) \leq \liminf _{n \rightarrow \infty}\left[F^{n}(x)-x\right] / n \leq \lim \sup _{n \rightarrow \infty}\left[F^{n}(x)-x\right] / n \leq M_{t}^{+}(z, f)$ for $x \in \pi^{-1}(z)$ and $r, t \in \mathbb{R}$, we may define the interval $M_{\alpha}(z, f)$, possibly degenerate, by $M_{\alpha}(z, f)=\left[M_{\alpha}^{-}(z, f), M_{\alpha}^{+}(z, f)\right]$. Observe that $\rho(z, f) \subset M_{\alpha}(z, f)$ for all $\alpha \in \mathbb{R}$ and $z \in S^{1}$. Clearly $M_{\alpha}^{-}$and $M_{\alpha}^{+}$satisfy the properties (R2) and (R3) for all $\alpha \in \mathbb{R}$. The next two lemmas show that indeed $M_{\alpha}^{-}$and $M_{\alpha}^{+}$are algorithms related to a rotation algorithm if and only if $\alpha \geq 1$.

LEMMA 2.3. Let $\alpha \in \mathbb{R}$. If one of the following statements

(a) $M_{\alpha}^{+}(z, f) \leq \rho^{+}(f)$ or

(b) $M_{\alpha}^{-}(z, f) \geq \rho^{-}(f)$

holds for every $f \in \operatorname{End}_{1}^{0}\left(S^{1}\right)$ and all $z \in S^{1}$ then $\alpha \geq 1$. 
Proof. Assume statement (a) holds for every $f \in \operatorname{End}_{1}^{0}\left(S^{1}\right)$ and all $z \in S^{1}$. In the proof we make use of a one-parameter family $\left\{F_{s}\right\}_{s \in(0,1)}$ of maps on $\mathbb{R}$. For $s \in(0,1)$ we define a map $F_{s}$ on $[0,1]$ as follows:

$$
F_{s}(0)=0, \quad F_{s}(s)=s+1, \quad F_{s}[(s+1) / 2]=s+2, \quad F_{s}(1)=1
$$

and on the intervals $[0, s],[s,(s+1) / 2]$ and $[(s+1) / 2,1]$ the map $F_{s}$ is linear. By putting $F_{s}(x+n)=F_{s}(x)+n$ for $n \in \mathbb{Z}$ and $x \in[0,1]$, the map $F_{s}$ is defined on $\mathbb{R}$. Then $F_{s}$ induces a circle map $f_{s} \in \operatorname{End}_{1}^{0}\left(S^{1}\right)$. We claim that $\rho^{+}\left(f_{s}\right)=1$ for $s \in(0,1)$.

In showing this we introduce a map $F_{s}^{+}, s \in(0,1)$ by

$$
\left.F_{s}^{+}\right|_{[0, s]} \equiv s+1,\left.\quad F_{s}^{+}\right|_{[s,(s+1) / 2]} \equiv F_{s} \quad \text { and }\left.\quad F_{s}^{+}\right|_{[(s+1) / 2,1]} \equiv s+2 \text {, }
$$

and further $F_{s}^{+}(x+n)=F_{s}^{+}(x)+n$ for $n \in \mathbb{Z}$ and $x \in[0,1]$. The map $F_{s}^{+}$defines a monotonic $f_{s}^{+} \in \operatorname{End}_{1}^{0}\left(S^{1}\right)$ and $F_{s} \leq F_{s}^{+}$so we have $\rho^{+}\left(f_{s}\right) \leq \rho\left(f_{s}^{+}\right)$. As $F_{s}^{k}(s)=$ $\left(F_{s}^{+}\right)^{k}(s), k \geq 0$, and $\rho\left(f_{s}^{+}\right)=1$ for all $s \in(0,1)$ we conclude that $\rho^{+}\left(f_{s}\right)=1$ for all $s \in(0,1)$. This proves the claim.

Now we finish the proof of the lemma. Because $f_{s} \in \operatorname{End}_{1}^{0}\left(S^{1}\right), s \in(0,1)$, we have by hypothesis

$$
\frac{s+3}{2}-\alpha=F_{s}\left(\frac{s+1}{2}\right)-\frac{s+1}{2}-\alpha \leq 1 \quad \text { for all } s \in(0,1) .
$$

This forces $\alpha \geq 1$.

The proof of the other case is analogous. So the lemma is proved.

LeMma 2.4. Let $f \in \operatorname{End}_{1}^{0}\left(S^{1}\right)$, then:

(i) $\alpha<\beta$ implies $M_{\alpha}(z, f) \supset M_{\beta}(z, f)$ for all $z \in S^{1}$.

(ii) $M_{1}(z, f) \subset \rho(f)$ for all $z \in S^{1}$.

Proof. The first statement is obvious. In case (ii) assume, by contradiction, there exists $z=\pi(x) \in S^{1}$ with $M_{1}^{+}(z, f)>\rho^{+}(f)$. Taking an iterate $F^{k}(x)$ of $x$ if necessary, we may assume that for certain $n \in \mathbb{N}$ :

$$
\frac{F^{n}(x)-x-1}{n}>\rho^{+}(f) \text {. }
$$

There also exists $y$ such that $F^{n}(y)-y \leq n \rho^{+}(f)$. So, by continuity of $F$, there is $\tilde{y}$ for which $F^{n}(\tilde{y})-\tilde{y}=k$, with $k$ an integer and $\left.k \in\left(n \rho^{+}(f), n \rho^{+}(f)\right)+1\right]$. Then $\pi(\tilde{y})$ is a periodic point of $f$ with rotation number equal to $k / n>\rho^{+}(f)$. Here we reach a contradiction.

Similarly one shows that $M_{1}^{-}(z, f)>\rho^{-}(f)$ for all $z \in S^{1}$. This proves the lemma.

We point out that neither $M^{-}$nor $M^{+}$need be constant on every orbit of an $f \in \operatorname{End}_{1}^{0}\left(S^{1}\right)$. For instance choose $f$ in such a way that a lift $F$ satisfies

$$
F(0)=\frac{1}{2}, \quad F\left(\frac{1}{4}\right)=2 \text { and } F\left(\frac{1}{2}\right)=1 .
$$

Then, by identifying $s \in[0,1)$ with its projection onto $S^{1}$, we have

$$
\frac{3}{4}=M^{+}\left(\frac{1}{4}, f\right)>M^{+}\left(f\left(\frac{1}{4}\right), f\right)=M^{+}(0, f)=\frac{1}{2} .
$$

By this same example it is also clear that $M_{\alpha}^{-}(X)$ is not necessarily smaller than 
$M_{\alpha}^{+}(X), X$ an orbit interval of $F$. For let $X=\left\{0, \frac{1}{2}, 1\right\}$ then

$$
M_{\alpha}^{-}(X)=\frac{1}{2}+\alpha / 2>M_{\alpha}^{+}(X)=\frac{1}{2}-\alpha / 2 \quad \text { for } \alpha \geq 1 .
$$

Notice that $M_{\alpha}^{-}(\operatorname{orb}(0, F))=M_{\alpha}^{+}(\operatorname{orb}(0, F))=\frac{1}{2}$.

We relate the numbers $M_{\alpha}^{-}(X)$ and $M_{\alpha}^{+}(X)$ to $M_{\alpha}(X)$ : if $M_{\alpha}^{-}(X)<M_{\alpha}^{+}(X)$ we put $M_{\alpha}(X)=\left[M_{\alpha}^{-}(X), M_{\alpha}^{+}(X)\right]$. For $1 \leq \alpha<\beta$ we would rather use the rotation algorithm $M_{\alpha}$ than $M_{\beta}$, see lemma 2.4(i). We formalize this by ordering the rotation algorithms with the relation $<$.

Let $A$ and $B$ be two rotation algorithms. We say $A$ is better than $B$, in notation $A<B$, if and only if $A^{-}(X) \leq B^{-}(X)$ and $B^{+}(X) \leq A^{+}(X)$ for each orbit interval $X$ and there exists an orbit interval for which one of the inequalities is strict.

Note that the relation $<$ does not define a total ordering: there exist algorithms $A$ and $B$ for which neither $A<B$ nor $B<A$. We have $M_{1}<M_{\alpha}$ for all $\alpha>1$. In view of lemma 2.3 one may wonder whether there is a better algorithm than $M=M_{1}$. In fact there is such an algorithm. This algorithm $S$, which we define below, is clearly the best two point rotation algorithm.

As before we begin by defining $S^{-}$and $S^{+}$. Let $Y=\left\{F^{n}(x)\right\}_{n=0}^{N}$ be an orbit interval, then $S^{-}(Y)$ and $S^{+}(Y)$ are given by

and

$$
S^{-}(Y)=\inf _{N \geq j>i \geq 0} \frac{\left[F^{j}(x)-F^{i}(x)\right]+1}{j-i}
$$

$$
S^{+}(Y)=\sup _{N \geq j>i \geq 0} \frac{\left[F^{j}(x)-F^{i}(x)\right]}{j-i} .
$$

Remark. Here $[a], a \in \mathbb{R}$, denotes the largest integer $n$ such that $n \leq a$.

Arguing in a similar way as we did in the case of the algorithm $M$, we conclude that $S^{-}$and $S^{+}$satisfy the properties (R1), (R2) and (R3). For $Y=$ orb $(x, F)$ with $\pi(x)=z$ we let

$$
S(z, f)=\left[S^{-}(Y), S^{+}(Y)\right] .
$$

The interval $S(z, f) \subset \rho(f)$ is called the speed interval of $z$ under $f$. The numbers $S^{-}(z, f)$ and $S^{+}(z, f)$ can be regarded as a modified minimum and maximum mean speed in orb $(x, F), z=\pi(x)$. Note that $S(z, f)$ is a topological invariant: if $h: S^{1} \rightarrow S^{1}$ is an orientation preserving homeomorphism then

$$
S(z, f)=S\left(h(z), h f h^{-1}\right) .
$$

Let $\operatorname{Hom}^{0}\left(S^{1}\right)$ be the set of continuous orientation preserving homeomorphisms of the circle.

LEMMA 2.5. Let $f \in \operatorname{End}_{1}^{0}\left(S^{1}\right)$, then for all $z \in S^{1}$ :

(i) $S^{-}(z, f)=\inf _{h \in \operatorname{Hom}^{0}\left(S^{1}\right)} M^{-}\left(h(z), h f h^{-1}\right)$;

(ii) $S^{+}(z, f)=\sup _{h \in \operatorname{Hom}^{0}\left(S^{1}\right)} M^{+}\left(h(z), h f h^{-1}\right)$

Proof. We only prove (i); the proof of (ii) is analogous. For $x \in \pi^{-1}(z)$ there exist two sequences $\{i(n)\}_{n \geq 0}$ and $\{j(n)\}_{n \geq 0}$ of positive integers such that

$$
\frac{\left[F^{j(n)}(x)-F^{i(n)}(x)\right]+1}{j(n)-i(n)}=S_{n}^{-}(z, f) \quad \downarrow \quad S^{-}(z, f) \quad \text { as } n \rightarrow \infty .
$$


For convenience we write $r(n)=j(n)-i(n)$. If $\{r(n)\}_{n \geq 1}$ is an unbounded sequence then $S^{-}(z, f)=M^{-}(z, f)$. We now consider the case $\{r(n)\}_{n \geq 0}$ is a bounded sequence. For $h \in \operatorname{Hom}^{0}\left(S^{1}\right)$ we have $S^{-}(z, f) \leq M^{-}\left(h(z), h f h^{-1}\right)$. Given $1>\varepsilon>0$ we shall construct $h_{\varepsilon} \in \operatorname{Hom}^{0}\left(S^{1}\right)$ such that

$$
M^{-}\left(h_{\varepsilon}(z), h_{\varepsilon} f h_{\varepsilon}^{-1}\right) \leq S^{-}(z, f)+\varepsilon .
$$

With this the proof of the lemma will be completed.

Because $S_{n}^{-}(z, f) \downarrow S^{-}(z, f)$ as $n \rightarrow \infty$, there exists $n_{0}$ such that

$$
S_{n}^{-}(z, f)<S^{-}(z, f)+\varepsilon / 2 \quad \text { for } n \geq n_{0} .
$$

Suppose $F^{j(m)}(x)=F^{i(m)}(x)+k(m)$ for some $m \geq n_{0}$, where $k(m)=$ $\left[F^{j(m)}(x)-F^{i(m)}(x)\right]$ and $\pi(x)=z$. Then $z$ is a periodic point of $f$ with rotation number $\rho(z, f)$ equal to $k(m) / r(m)$ and $S_{n}^{-}(z, f)=\rho(z, f)+(1 / r(m))$. Since $\{r(n)\}_{n \geq 0}$ is a bounded sequence and $\rho(z, f) \subset S(z, f)$, we conclude that

$$
F^{j(n)}(x) \neq F^{i(n)}(x)+k(n) \quad \text { for some } n \geq n_{0} .
$$

Assume $F^{j(n)}(x)>F^{i(n)}(x)+k(n)$ and thus

$$
F^{i(n)}(x)<F^{j(n)}(x)-k(n)<F^{i(n)}(x)+1 .
$$

Define a map $H_{\varepsilon}$ on $\mathbb{R}$ as follows:

Choose $H_{\varepsilon}\left(F^{i(n)}(x)\right)$ arbitrarily and let

$$
H_{\varepsilon}\left(F^{i(n)}(x)+m\right)=H_{\varepsilon}\left(F^{i(n)}(x)+m\right.
$$

and

$$
H_{\varepsilon}\left(F^{j(n)}(x)-k(n)+m\right)=H_{\varepsilon}\left(F^{i(n)}(x)+\varepsilon / 2+m\right.
$$

for all $m \in \mathbb{Z}$. Further $H_{\varepsilon}$ is piecewise linear.

Then the map $H_{\varepsilon}$ induces $h_{\varepsilon} \in \operatorname{Hom}^{0}\left(S^{1}\right)$ with the desired properties. For we have

$$
\begin{aligned}
M^{-}\left(h_{\varepsilon}(z), h_{\varepsilon} f h_{\varepsilon}^{-1}\right) & \leq \frac{H_{\varepsilon}\left(F^{j(n)}(x)\right)-H_{\varepsilon}\left(F^{i(n)}(x)\right)+1}{j(n)-i(n)} \\
& =\frac{k(n)+1+\varepsilon / 2}{r(n)} \leq S_{n}^{-}(z, f)+\varepsilon / 2<S^{-}(z, f)+\varepsilon .
\end{aligned}
$$

The case $F^{j(n)}(x)<F^{i(n)}(x)+k(n)$ can be handled in a similar way. So we are done.

\section{Interpolation maps}

Let $F$ be a lift of $f \in \operatorname{End}_{1}^{0}\left(S^{1}\right)$. In [8] the following maps on $\mathbb{R}$ are introduced (see also figure 3.1 ):

$$
F^{-}(x)=\inf _{y \geq x} F(y) \text { and } F^{+}(x)=\sup _{y \leq x} F(y) .
$$

Both maps $F^{-}$and $F^{+}$are continuous and induce monotone circle maps of degree one, $f^{-}$and $f^{+}$respectively. The map $F^{-}$may be characterized as the largest non-decreasing map less than or equal to $F$. Likewise $F^{+}$may be characterized as the smallest non-decreasing map greater than or equal to $F$. Since $f^{-}$and $f^{+}$are monotone their rotation number is properly defined and we have the following known result, cf. [8], [5]. 


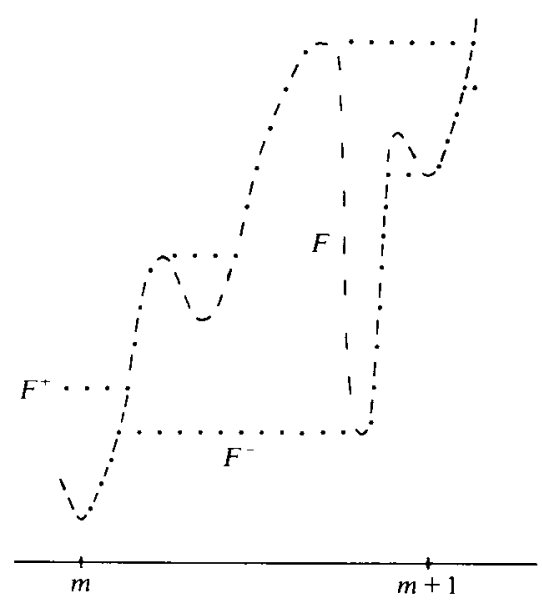

FIGURE 3.1

Lemma 3.1. Let $f \in \operatorname{End}_{1}^{0}\left(S^{1}\right)$ then $\rho\left(f^{-}\right)=\rho^{-}(f)$ and $\rho\left(f^{+}\right)=\rho^{+}(f)$.

Proof. We first prove that $\rho\left(f^{-}\right)=\rho^{-}(f)$. As $F^{-}(x) \leq F(x)$ for all $x \in \mathbb{R}$ and $F^{-}$is non-decreasing, we have $\left(F^{-}\right)^{n}(x) \leq F^{n}(x)$ for all $x \in \mathbb{R}$ and all $n \geq 0$. This implies that $\rho\left(f^{-}\right) \leq \rho^{-}(f)$. Assume $\rho\left(f^{-}\right) \leq \rho^{-}(f)$, then there exists a rational $p / q$ such that $\rho\left(f^{-}\right)<p / q<\rho^{-}(f)$ and consequently $F^{q}(x)>x+p$ for all $x \in \mathbb{R}$. Now choose $y_{0} \in \mathbb{R}$ and define a sequence $\left\{y_{i}\right\}_{i=1}^{q}$ by

$$
y_{i+1}=\sup \left\{F^{-1}\left(y_{i}\right)\right\} \quad \text { for } i=0, \ldots, q-1 .
$$

In that case $F^{-}\left(y_{i+1}\right)=F\left(y_{i+1}\right)=y_{i}, 0 \leq i \leq q-1$, and thus $\left(F^{-}\right)^{q}\left(y_{q}\right)=F^{q}\left(y_{q}\right)>$ $y_{q}+p$. So $\rho\left(f^{-}\right) \geq p / q$ and we have a contradiction. Hence $\rho\left(f^{-}\right)=\rho^{-}(f)$; the proof that $\rho\left(f^{+}\right)=\rho(f)$ is similar.

The construction of a homotopy from $F^{-}$to $F^{+}$, as given in [5], can be generalized, resulting in a continuous homotopy for arbitrary $f \in \operatorname{End}_{1}^{0}\left(S^{1}\right)$, (see also [6] where a homotopy is given similar to that we present below). We shall define for $f \in$ $\operatorname{End}_{1}^{0}\left(S^{1}\right)$ a continuous family $\left\{\Phi_{\mu}\right\}_{\mu \in[0,1]}$ of continuous non-decreasing maps on $\mathbb{R}$ such that $\Phi_{0} \equiv F^{-}$and $\Phi_{1} \equiv F^{+}$. This family $\left\{\Phi_{\mu}\right\}_{\mu \in[0,1]}$ we then employ in defining a two-parameter family. For each interval $I \subset \rho(f)$, possibly degenerate to a point, there belongs a map to this family such that the corresponding endomorphism of the circle has a rotation interval equal to $I$. Such a map we shall call an interpolation map.

In defining $\left\{\Phi_{\mu}\right\}_{\mu \in[0,1]}$ we first consider the case $f \in \operatorname{End}_{1}^{0}\left(S^{1}\right)$ has two critical points. At a critical point $f$ is not locally a homeomorphism. The set of critical points of $f$ we denote by $C(f)$.

Let $F$ assume a local minimum at $m$. The remaining extremum of $F$ in $(m, m+1)$, where $F$ has a local maximum, we denote by $M$. We make use of the continuous map $\widetilde{F^{+}}$given by

$$
\widetilde{F^{+}}(x)=\sup _{m \leq y \leq x} F(y) \quad \text { for } x \in[m, m+1] \text {, see figure } 3.2 .
$$


Further let $t(\mu), \mu \in[0,1]$, be the smallest value in $[m, M]$ for which

$$
F(t(\mu))=(1-\mu) F(m)+\mu F(M) \text {. }
$$

Then the family $\left\{\Phi_{\mu}\right\}_{\mu \in[0,1]}$ is defined on $[m, m+1]$ as follows:

$$
\Phi_{\mu}(x)=\left\{\begin{array}{l}
\max \left[F(t(\mu))-1, \widetilde{F^{+}}(x)\right], \quad x \in[m, t(\mu)] \\
\max \left[F(t(\mu)), F^{-}(x)\right], \quad x \in((t)(\mu), m+1]
\end{array} \quad \text { for } \mu \in[0,1] .\right.
$$

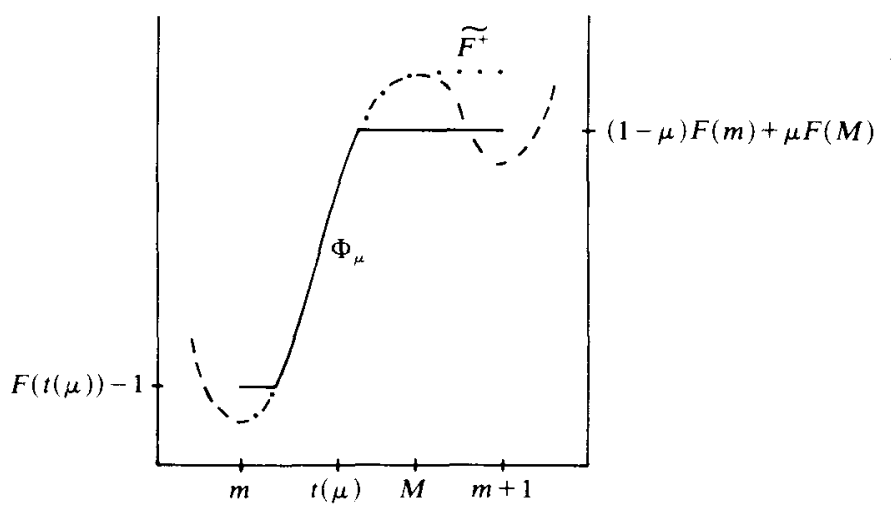

FIGURE 3.2

For $f$ with $\# C(f)=2$ it is easily verified that $\Phi_{0} \equiv F^{-}$and $\Phi_{1} \equiv F^{+}$on $[m, m+1]$. We point out that $\Phi_{\mu}$ is defined in such a way as to make the definition easily adaptable to general circle maps. If $\# C(f)=2$, replacing $\widetilde{F}^{+}$by $F$ in the above formula results in the same $\Phi_{\mu}$ for all $\mu$.

Before an example is given where we really need $\widetilde{F^{+}}$, we explain how to interpret the definition of $\Phi_{\mu}$ for a general circle map $f$. We only have to make some minor adjustments. Again let $m$ and $M$ be extrema of $F$ but such that $F$ has a local minimum at $m$ with $F^{-}(m)=F(m)$ and $F$ assumes its maximum on $[m, m+1]$ at $M$ where $M$ is chosen minimal. The rest as defined above remains unaltered. See figure 3.3 for an illustration of this procedure in the general case. Notice the rôle of $\widetilde{F^{+}}$in making $\Phi_{\mu}$ a non-decreasing map.

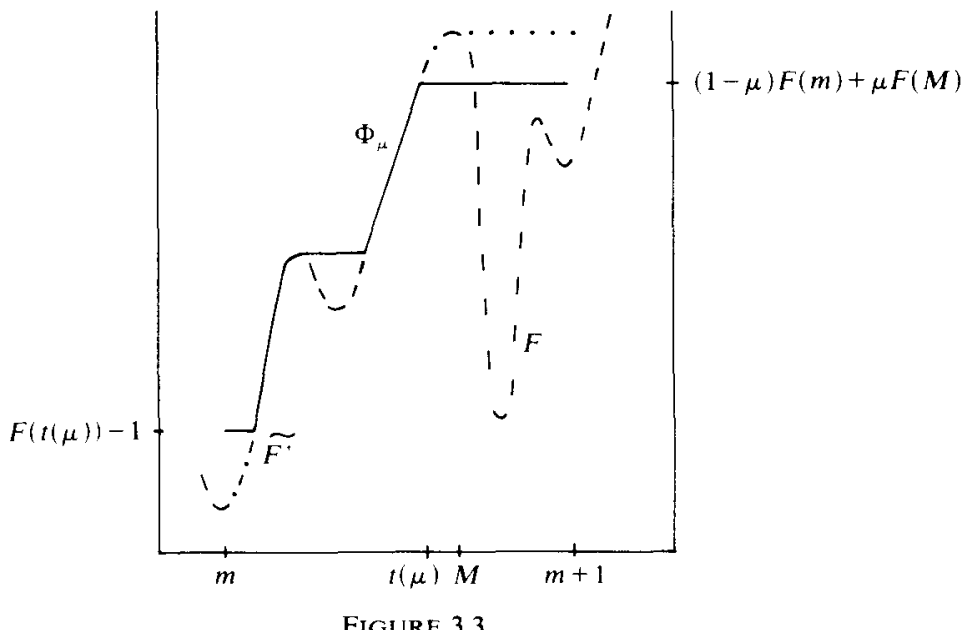

FIGURE 3.3 
As $\Phi_{\mu}(m+1)=\max \left[F(t(\mu)), F^{-}(m+1)\right]=\max [F(t(\mu)), F(m)+1]=\Phi_{\mu}(m)+1$, we may extend $\Phi_{\mu}$ to a map on $\mathbb{R}$, which we also denote by $\Phi_{\mu}$, by the identity

$$
\Phi_{\mu}(x+n)=\Phi_{\mu}(x)+n \quad \text { for all } n \in \mathbb{Z} \quad \text { and } \quad x \in[m, m+1] .
$$

The map $\Phi_{\mu}$ induces a circle map $\varphi_{\mu}$. In listing some properties of these maps we use the following notion:

Let $G: \mathbb{R} \rightarrow \mathbb{R}$. An open and maximal interval on which $G$ is constant is called a plateau of $G$. The union of all plateaus of $G$ is denoted by $\operatorname{P} 1(G)$.

LEMMA 3.2. (i) The family $\left\{\Phi_{\mu}\right\}_{\mu \in[0,1]}$ is continuous, $\varphi_{\mu} \in \operatorname{End}_{1}^{0}\left(S^{1}\right)$ and $\varphi_{\mu}$ is monotonic for $\mu \in[0,1]$.

(ii) If $0 \leq a<b \leq 1$ then $\Phi_{a} \leq \Phi_{b}$. Further $\Phi_{0} \equiv F^{-}$and $\Phi_{1} \equiv F^{+}$.

(ii) Let $W$ be an interval such that $W \cap P 1\left(\Phi_{\mu}\right)=\varnothing$ then $\Phi_{\mu} \equiv F$ on $W, \mu \in[0,1]$.

Proof. (i) We first consider the continuity of $\left\{\Phi_{\mu}\right\}_{\mu \in[0,1]}$. Fix $\mu_{0} \in[0,1]$. For a given $\varepsilon<1$ there exists a neighbourhood $V \subset[0,1]$ of $\mu_{0}$ such that $\left|F\left(t\left(\mu_{0}\right)\right)-F(t(\mu))\right|<\varepsilon$ for all $\mu \in V$. For a $\mu \in V$ different from $\mu_{0}$ we may assume that $t\left(\mu_{0}\right)<t(\mu)$; in case $t\left(\mu_{0}\right)>t(\mu)$ the proof is analogous. It is a direct consequence of the definition of $\Phi_{\mu}$ that

$$
\left|\Phi_{\mu}(x)-\Phi_{\mu_{0}}(x)\right|<\varepsilon \quad \text { for } x \in\left[m, t\left(\mu_{0}\right)\right] \cup[t(\mu), m+1]
$$

The same inequality holds for $x \in\left[t\left(\mu_{0}\right), t(\mu)\right]$. One easily checks that $\widetilde{F^{+}}(t(\mu))=$ $F(t(\mu))$ for $\mu \in[0,1]$ and thus $\widetilde{F^{+}}(x) \geq F\left(t\left(\mu_{0}\right)\right)$ for $x \in\left[t\left(\mu_{0}\right), m+1\right]$. Further $F\left(t\left(\mu_{0}\right)\right)>F(t(\mu))-1$ because $\varepsilon<1$, so we have

$$
F\left(t\left(\mu_{0}\right)\right) \leq \Phi_{\mu}(x) \leq F(t(\mu)) \quad \text { for } x \in\left[t\left(\mu_{0}\right), t(\mu)\right] .
$$

If $x \in[m, t(\mu)]$ then $F^{-}(x) \leq F(x) \leq F(t(\mu))$ and thus

$$
F\left(t\left(\mu_{0}\right)\right) \leq \Phi_{\mu_{0}}(x) \leq F(t(\mu)) \quad \text { for } x \in\left[t\left(\mu_{0}\right), t(\mu)\right] .
$$

We conclude that $\max _{x \in \mathbb{R}}\left|\Phi_{\mu}(x)-\Phi_{\mu_{0}}(x)\right|<\varepsilon$ for all $\mu \in V$. Since $\varepsilon<1$ was arbitrary, we thus have proved the continuity of $\left\{\Phi_{\mu}\right\}_{\mu \in[0,1]}$.

Since $\lim _{x \uparrow m+1} \Phi_{\mu}(x)=\lim _{x \downarrow m} \Phi_{\mu}(x)+1$ it suffices, in proving the continuity of $\varphi_{\mu}$, to establish the continuity of $\Phi_{\mu}$ on $[m, m+1]$. We have $\widetilde{F}^{+}(t(\mu))=F(t(\mu))$ and $F^{-}(t(\mu))<F(t(\mu))$, so the map $\Phi_{\mu}$ is continuous at $t(\mu)$. The continuity on $[m, t(\mu)]$ or $[t(\mu), m+1]$ is clearly guaranteed. Therefore $\varphi_{\mu} \in \operatorname{End}_{1}^{0}\left(S^{1}\right)$ and the monotonicity of $\varphi_{\mu}$ follows immediately from the properties of $\widetilde{F^{+}}$and $F^{-}$.

(ii) Let $0 \leq a<b \leq 1$ be given. In that case $t(a)<t(b)$ and $F(t(a))<F(t(b))$. So we have

$$
\Phi_{a}(x) \leq \Phi_{b}(x) \quad \text { for } x \in[m, t(a)] \cup[t(b), m+1] .
$$

If $x \in[t(a), m+1]$ then $\widetilde{F^{+}}(x) \geq F(t(a))$ and thus

$$
\begin{aligned}
\Phi_{a}(x) & =\max \left[F(t(a)), F^{-}(x)\right] \leq \max \left[F(t(a)), \widetilde{F^{+}}(x)\right] \\
& \leq \widetilde{F^{+}}(x) \leq \max \left[F(t(b))-1, \widetilde{F^{+}}(x)\right] \\
& =\Phi_{b}(x) \quad \text { for } x \in[t(a), t(b)] .
\end{aligned}
$$

We conclude that $\Phi_{a} \leq \Phi_{b}$ on $[m, m+1]$ and consequently $\Phi_{a} \leq \Phi_{b}$ on $\mathbb{R}$. Further $\Phi_{0} \equiv F^{-}$and $\Phi_{1} \equiv F^{+}$because $t(0)=m$ and $t(1)=M$. 
(iii) Fix $\mu \in[0,1]$. As $\Phi_{\mu}(x+1)=\Phi_{\mu}(x)+1$ for all $x \in \mathbb{R}$, we may assume that $W \subset[m, m+1]$. Let $x \in W$ and suppose that $x \in[m, t(\mu)]$. Since $x \notin \operatorname{Pl}\left(\Phi_{\mu}\right)$ we have that $\Phi_{\mu}(x)=\widetilde{F}^{+}(x)$. Assume that $F(x)<\widetilde{F^{+}}(x)$, then there exists $y$ with $m \leq y \leq x$ and a neighbourhood $U$ of $x$ such that $\widetilde{F^{+}} \equiv F(y)$ on $U$. Here we reach a contradiction and thus $\Phi_{\mu}(x)=F(x)$. In a similar way one proves that $\Phi_{\mu}(x)=F(x)$ for $x \in[t(\mu), m+1] \cap W$. This finishes the proof of the lemma.

Now we have a smooth homotopy $\left\{\Phi_{\mu}\right\}_{\mu \in[0,1]}$ from $F^{-}$to $F^{+}$, we proceed with the construction of the so-called interpolation maps of $f$. In what follows we define for any given interval $I \subset \rho(f)$, possibly degenerate, a map $H$ which equals $F$ except for a set of intervals where it is constant and which induces a circle map $h \in \operatorname{End}_{1}^{0}\left(S^{1}\right)$ with $\rho(h)=I$.

Since $\left\{\varphi_{\mu}\right\}_{\mu \in[0,1]}$ is a continuous family of monotone maps, $\varphi_{\mu}$ admits a rotation number $\rho\left(\varphi_{\mu}\right)$ and $\rho\left(\varphi_{\mu}\right)$ depends continuously on $\mu$, cf. [3]. So given an interval $I \subset \rho(f)$ there exist according to lemma 3.1 and lemma 3.2(ii) numbers $a$ and $b$, $0 \leq a \leq b \leq 1$, such that

$$
\left[\rho\left(\varphi_{a}\right), \rho\left(\varphi_{b}\right)\right]=I .
$$

We remark that instead of using lemma 3.1 here, it suffices to observe that $\rho\left(f^{-}\right) \leq$ $\rho^{-}(f)$ and $\rho\left(f^{+}\right) \geq \rho^{+}(f)$.

In case $I$ is a singleton we define $H=H_{a}$ equal to $\Phi_{a}$.

If $I$ is non-degenerate then $\Phi_{a}$ and $\Phi_{b}$ are not identical. By lemma 3.2(iii) there exists a set $\left\{S_{j}\right\}_{j \in J}$ of disjunct open intervals so that on each interval $S_{j}$ both $\Phi_{a}$ and $\Phi_{b}$ are constant. The intervals $S_{j}$ are chosen maximal. We now define $H=H_{a, b}$ as follows (see also figure 3.4 ):

(i) on $\mathbb{R} \backslash \bigcup_{j} S_{j}$ the map $H_{a, b}$ is equal to $F$.

Write $S_{j}=\left[a_{j}, b_{j}\right], j \in I$, and define

$$
p_{j}=\max \left\{x \in S_{j} \mid F(x)=F\left(a_{j}\right)\right\}
$$

and

$$
q_{j}=\min \left\{x \in\left[p_{j}, b_{j}\right] \mid F(x)=F\left(b_{j}\right)\right\} .
$$

(ii) On $\left[p_{j}, q_{j}\right], j \in J$, the map $H_{a, b}$ is also equal to $F$.

(iii) On an interval $K=(u, v)$ where $H_{a, b}$ is not yet defined, $H_{a, b}$ is constant: $H_{a, b} \equiv H_{a, b}(u)$ on $K$.

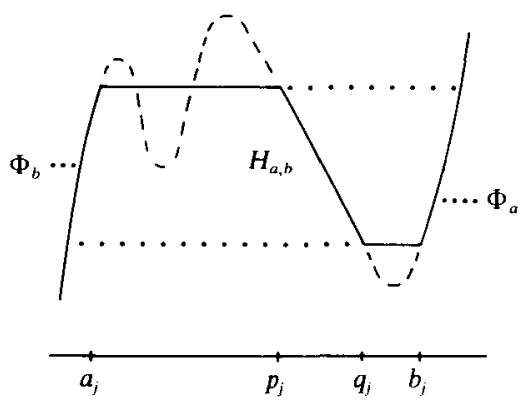

FIGURE 3.4 
A map $H_{a, b}$ with $0 \leq a \leq b \leq 1$ as defined above is called an interpolation map of $f$. Observe that $h_{a, b} \in \operatorname{End}_{1}^{0}\left(S^{1}\right)$. We also have $\rho\left(h_{a, b}\right)=I$ according to the next lemma.

LeMMA 3.3. The map $H_{a, b}$ satisfies $H_{a, b}^{-} \equiv \Phi_{a}$ and $H_{a, b}^{+} \equiv \Phi_{b}$.

Proof. Because of the construction of $H_{a, b}$ we have $\Phi_{a} \leq H_{a, b} \leq \Phi_{b}$ and thus $\Phi_{a} \leq H_{a, b}^{-} \leq H_{a, b}^{+} \leq \Phi_{b}$. We prove that $H_{a, b}^{-} \equiv \Phi_{a}$. Assume, by contradiction, that there exists $p$ such that $H_{a, b}^{-}(p)>\Phi_{a}(p)$. Let $V$ be the maximal interval containing $p$ on which $H_{a, b}^{-}>\Phi_{a}$. This interval is bounded and we write $V=(c, d)$. Then $\Phi_{a}(c)<\Phi_{a}(d)$ since both $\Phi_{a}$ and $H_{a, b}^{-}$are non-decreasing maps. So there is an interval $W=[u, v]$ contained in $V$ such that $\Phi_{a} \equiv H_{a, b}$ on $W$ and thus

$$
H_{a, b}^{-}(u)=\inf _{x \geq u} H_{a, b}(x) \leq H_{a, b}(u)=\Phi_{a}(u) .
$$

Here we reach a contradiction and we conclude that $H_{a, b}^{-} \equiv \Phi_{a}$. The proof that $H_{a, b}^{+} \equiv \Phi_{b}$ is similar.

Remark. One can easily think of an example for which $H_{0,1}$ is not equal to $F$ (see figure 3.5).

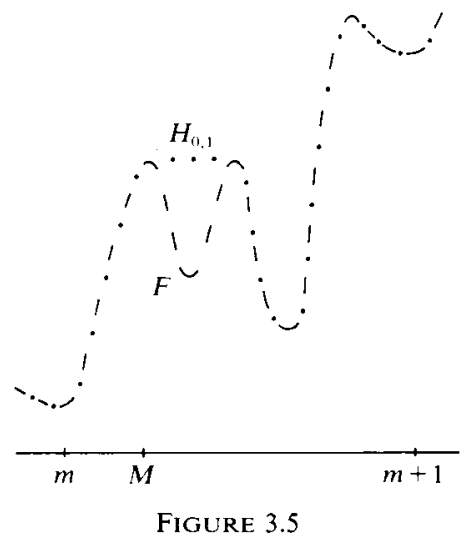

LEMMA 3.4. Let $H_{a, b}$ be an interpolation map of $f$. If $x \in \operatorname{PI}\left(H_{a, b}\right)$ then $x \in \operatorname{Pl}\left(\Phi_{s}\right)$ for all $s \in[a, b]$.

Proof. Suppose $x \notin \mathrm{Pl}\left(\Phi_{s}\right)$ for some $s \in[a, b]$. We may assume that $x \in[m, m+1]$. Since $x \notin \operatorname{Pl}\left(\Phi_{s}\right)$ there exists an interval $U$ with $x \in U$ such that $U \cap \operatorname{Pl}\left(\Phi_{s}\right)=\varnothing$. We can choose $U$ so small that $t(s) \notin$ int $U$. If $t(s)$ is on the left side of $U$ then $U \cap \operatorname{Pl}\left(\Phi_{a}\right)=\varnothing$. So we have $x \notin \operatorname{Pl}\left(H_{a, b}\right)$. The same conclusion follows if $t(s)$ is on the right side of $U$. For in that case $U \cap \mathrm{Pl}\left(\Phi_{b}\right)=\varnothing$. This proves the lemma.

\section{Linking of sequences}

The family $\left\{\Phi_{\mu}\right\}_{\mu \in[0,1]}$ enables us to prove, in an easy way, the existence of particularly simple periodic orbits. The periodic orbits which we are aiming at, are usually referred to as twist periodic orbits, cf. [1].

Let $S$ be a periodic orbit of $f \in \operatorname{End}_{1}^{0}\left(S^{1}\right)$ with rotation number $p / q,(p, q)=1$. The orbit $S$ is called a twist periodic orbit of $f$ if $f$ is strictly monotone on $S$, i.e. $x<y$ implies $F(x)<F(y)$ for all $x, y \in \pi^{-1}(S)$. 
LEMMA 4.1 Let $S$ be a twist periodic orbit of $f \in \operatorname{End}_{1}^{0}\left(S^{1}\right)$ with rotation number $p / q$, $q \geq 1$, and $\pi^{-1}(S)=\left\{x_{i}\right\}_{i \in \mathbf{Z}}$ where $x_{i}<x_{i+1}$ for all $i$, then:

(i) the orbit $S$ has period $q$;

(ii) $F\left(x_{i}\right)=x_{i+p}$ for all $i$.

Proof. Since $S$ has rotation number $p / q$ with $(p, q)=1$ and $q \geq 1$, the period of $S$ is a multiple of $q$, say $m q$ with $m \geq 1$. This implies that $x_{i+k m q}=x_{i}+k$ for all $i, k \in \mathbb{Z}$. Moreover $S$ is a twist periodic orbit and $F$ on $\pi^{-1}(S)$ is one-to-one, so we have $F\left(x_{i}\right)=x_{i+r}$ for some $r \in \mathbb{Z}$ and all $i$. From this we conclude that

$$
x_{i}+m p=F^{m q}\left(x_{i}\right)=x_{i+r m q}=x_{i}+r \text {. }
$$

Hence $r=m p$ and $F^{q}\left(x_{i}\right)=x_{i+p m q}=x_{i}+p$. As $S$ is not the union of several orbits, $S$ has period $q$ and $F\left(x_{i}\right)=x_{i+p}$ for all $i$.

Twist periodic orbits just characterize the notion of a simple orbit: the order of a twist periodic orbit with rotation number $p / q$ around the circle is the same as that of an orbit of a monotone circle map with rotation number $p / q$. The next lemma assures the existence of twist periodic orbits. The same result was obtained in [1], but in a different way; see also [6].

Lemma 4.2. Let $f \in \operatorname{End}_{1}^{0}\left(S^{1}\right)$. For $p / q \in \rho(f)$ with $(p, q)=1$, there exists a twist periodic orbit of $f$ with rotation number $p / q$.

Proof. Clearly every fixed point of $f$ is a twist periodic orbit of $f$ so we may assume that $q \geq 2$. We make use of the family $\left\{\varphi_{\mu}\right\}_{\mu \in[0,1]}$ of monotone circle maps. Since $p / q \in \rho(f)$ we have $\rho\left(\varphi_{\mu}\right)=p / q$ for some $\mu \in[0,1]$. The map $\varphi_{\mu}$ has a periodic point $z$ with rotation number $p / q$. If orb $\left(x, \Phi_{\mu}\right)$ with $x \in \pi^{-1}(z)$ avoids $\operatorname{Pl}\left(\varphi_{\mu}\right)$ we are done, see lemma 3.2(iii). Suppose on the contrary that there exists $x_{0} \in$ orb $\left(x, \Phi_{\mu}\right)$ such that $x_{0}$ belongs to a plateau $V$ of $\Phi_{\mu}$. Let $V=(a, b)$ and thus $\Phi_{\mu}^{q}(a)>a+p$ and $\Phi_{\mu}^{q}(b)<b+p$. Define $y$ as follows:

$$
y=\min _{u \geq b}\left\{u \mid \Phi_{\mu}^{q}(u)=u+p\right\} .
$$

Since $\varphi_{\mu} \in \operatorname{End}_{1}^{0}\left(S^{1}\right)$ the point $y$ is well defined and $y \notin \operatorname{Pl}\left(\Phi_{\mu}^{q}\right)$. So we have $\operatorname{orb}\left(y, \Phi_{\mu}\right) \cap \operatorname{Pl}\left(\Phi_{\mu}\right)=\varnothing$ and $\pi(y)$ is a period point of $f$ with rotation number $p / q$. This proves the lemma.

We showed the existence of a twist periodic orbit, say $S$, by means of the map $\Phi=\Phi_{\mu}$. Next we construct, for a given $N \in \mathbb{N}$, closed subsets $K \subset \mathbb{R}$ such that $\left\{f^{i}(z)\right\}_{i=0}^{N}$, with $z \in \pi(K)$, is ordered around the circle in the same way as $S$.

Let $N \in \mathbb{N}$ be given. Suppose $S$ has rotation number $p / q$ with $q \geq 2$. We write $\pi^{-1}(S)=\left\{x_{i}\right\}_{i \in \mathbb{Z}}$, where $x_{i}<x_{i+1}$ for all $i$. In defining $K$ we need two consecutive elements of $\pi^{-1}(S)$; we may take $x_{0}$ and $x_{1}$ for these. Since $\left.\Phi^{m}\left[x_{0}, x_{1}\right]\right)=\left[x_{m p}, x_{m p+1}\right]$ for $m \geq 0$ and $\pi^{-1}(S) \cap \mathrm{Pl}(\Phi)=\varnothing$ there exists $K \subset\left[x_{0}, x_{1}\right]$ for which:
(a) $\Phi^{i}(K) \cap \operatorname{PI}(\Phi)=\varnothing \quad$ for $i=0, \ldots, N-1$;
(b) $\Phi^{i}(K) \subset\left[x_{i p}, x_{i p+1}\right]$ for $i=0, \ldots, N$;
(c) $\Phi^{N}(K)=\left[x_{N p}, x_{N p+1}\right]$.

Then $K$ has the desired properties. We also have $F^{i}(K)=\Phi^{i}(K)=H_{a, b}^{i}(K)$ for 
$i=0, \ldots, N$ and $0 \leq a \leq \mu \leq b \leq 1$, see lemma 3.2(iii) and lemma 3.4. Let $K^{i}=$ $F^{i}(K)$. The sequence $\left\{K^{i}\right\}_{i=0}^{N}$ is said to be related to the twist periodic orbit $S$. If confusion is unlikely we sometimes write $\left\{U^{i}\right\}_{i=0}^{M} \sim(r / s)$ where $r / s$ is the rotation number of the twist periodic orbit to which $\left\{U^{i}\right\}_{i=0}^{M}$ is related. Note that for a given twist periodic orbit there are several sequences $\left\{K^{i}\right\}_{i=0}^{N}$ related to it.

LEMMA 4.3. Let $\left\{K^{i}\right\}_{i=0}^{N}$ be a sequence related to a twist periodic orbit $S$ off $\in \operatorname{End}_{1}^{0}\left(S^{1}\right)$ with rotation number $p / q$. Given $\varepsilon>0$, there exists $n_{0} \geq 0$ such that if $y \in K^{0}$ and $N \geq n_{0}$ then

$$
\left|\frac{F^{m}(y)-y}{m}-\frac{p}{q}\right|<\varepsilon \quad \text { with } n_{0} \leq m \leq N .
$$

Proof. Let $K^{0} \subset\left[x_{0}, x_{1}\right]$ with $x_{0}$ and $x_{1}$ two consecutive elements of $\pi^{-1}(S)$. Since $S$ has rotation number $p / q$ there exists a natural number $n_{0}$ such that

$$
\left|\frac{F^{n}\left(x_{0}\right)-x_{0}}{n}-\frac{p}{q}\right|<\frac{\varepsilon}{2} \quad \text { for } n \geq n_{0} .
$$

We may assume that $n_{0}$ is chosen so large that $2 / n_{0}<\varepsilon / 2$. Then we have

$$
\begin{aligned}
\left|\frac{F^{m}(y)-y}{m}-\frac{p}{q}\right| & =\left|\frac{F^{m}(y)-F^{m}\left(x_{0}\right)+x_{0}-y+F^{m}\left(x_{0}\right)-x_{0}}{m}-\frac{p}{q}\right| \\
& \leq \frac{2}{m}+\left|\frac{F^{m}\left(x_{0}\right)-x_{0}}{m}-\frac{p}{q}\right|<\varepsilon
\end{aligned}
$$

for $n_{0} \leq m \leq N$ and $y \in K^{0}$.

LemmA 4.4. Let $f \in \operatorname{End}_{1}^{0}\left(S^{1}\right)$. Given $m \in \mathbb{N}$ and $\varepsilon>0$ there exists $n_{0}$ such that for all $n \geq n_{0}$

$$
\left|\frac{F^{n+k}(x)-x}{n+k}-\frac{F^{n}(x)-x}{n}\right|<\varepsilon \quad \text { for all } x \in \mathbb{R}, \text { where } 0 \leq k \leq m .
$$

Proof. (See [4].)

In the last part of this section we discuss the possibility to transit from one sequence to another. By linking the appropriate sequences, a procedure which we make precise below, we are able to select $z \in S^{1}$ with the proper rotation interval and speed interval.

Suppose we have a sequence $\left\{U^{i}\right\}_{i=0}^{L}$ which is related to a twist periodic orbit $S$ with rotation number $p / q$. Let $T$ be another twist periodic orbit with rotation number $r / s$. If $s>q$ there exist two consecutive elements $x$ and $y$ of $\pi^{-1}(S)$ for which the set $[x, y] \cap \pi^{-1}(T)$ contains at least two elements. There exists $V^{0} \subset U^{0}$ for which the sequence $\left\{V^{i}\right\}_{i=0}^{M}$ is related to $S$ and such that $\pi\left(V^{M}\right)=\pi([x, y])$. Notice that $M$ can be chosen arbitrarily large. Then by choosing a suitable $W^{0} \subset V^{0}$ we get a sequence $\left\{W^{i}\right\}_{i=0}^{N}$ such that $W^{i} \subset V^{i}$ for $i=0, \ldots, M$ and $\left\{W^{i}\right\}_{i=M}^{N}$ is related to $T$. We say that $\left\{V^{i}\right\}_{i=0}^{M}$ is linked to a sequence which is related to $T$.

Remark. In its turn $\left\{\boldsymbol{W}^{i}\right\}_{i=0}^{N}$ can be linked to a sequence which is related to a twist periodic orbit $R$ provided $R$ has period $t$ with $t>r$. 


\section{Proof of the theorem}

We first assume that $I$ is non-degenerate. Since $I \subset J$ we thus have $I=[\alpha, \beta], \alpha<\beta$, and $J=[c, d], c<d$. Choose $\left\{\rho_{i}=p_{i} / q_{i}\right\}_{i \in \mathbb{N}}$ and $\left\{\sigma_{i}=r_{i} / s_{i}\right\}_{i \in \mathbb{N}}$ such that:

(a) $\alpha<\rho_{i}<\beta$ with $\left(p_{i}, q_{i}\right)=1$ and $c<\sigma_{i}<d$ with $\left(r_{i}, s_{i}\right)=1$ for $i \geq 1$;

(b) $\lim _{k \rightarrow \infty} \rho_{2 k+1}=\alpha$ and $\lim _{k \rightarrow \infty} \rho_{2 k}=\beta$;

(c) $\lim _{k \rightarrow \infty} \sigma_{2 k+1}=c$ and $\lim _{k \rightarrow \infty} \sigma_{2 k}=d$;

(d) $2 \leq q_{i}<s_{i}<q_{i+1}$ for $i \geq 1$.

We include (d) so that linking is possible. Let $\left\{\varepsilon_{i}\right\}_{i \in \mathbb{N}}$ be a sequence of positive real numbers such that

$$
\left[\rho_{i}-\varepsilon_{i}, \rho_{i}+\varepsilon_{i}\right] \subset[\alpha, \beta] \text { and }\left[\sigma_{i}-\varepsilon_{i}, \sigma_{i}+\varepsilon_{i}\right] \subset[c, d] \text { for } i \geq 1 .
$$

In the following we shall construct, by induction, for each $i \in \mathbb{N}$ a sequence $X_{i}=\left\{K_{i}^{m}\right\}_{m=0}^{N_{i}}$, where $\left\{K_{i}^{m}\right\}_{m=M_{i}}^{N_{i}} \sim\left(\rho_{i}\right)$ and $M_{1}=0$. Then $z \in \pi\left(\bigcap_{i \geq 1} K_{i}^{0}\right) \subset S^{1}$ will satisfy $\rho(z, f)=[\alpha, \beta]$ and $S(z, f)=[c, d]$.

Suppose $X_{i}$ is given for some $i \geq 1$. In determining $X_{i+1}$ we successively construct $X_{i, l}$ with $l=1,2$. Here $X_{i, l}$ is obtained by linking $X_{i, l-1}$ to a certain sequence and $X_{i, 0}$ is equal to $X_{\mathrm{i}}$. Notice that we assume that $X_{i, l}$ is suitable for linking. We remark that this assumption can be fulfilled inductively. In defining $X_{i+1}$ we distinguish two steps:

Step I. Link $X_{i}$ to a sequence which is related to a twist periodic orbit with rotation number $\sigma_{i}$. This results in a sequence $X_{i, 1}=\left\{K_{i, 1}^{m}\right\}_{m=0}^{M_{i+1}}$, where $\left\{K_{i, 1}^{m}\right\}_{m=N_{i}}^{M_{i+1}} \sim\left(\sigma_{i}\right)$, which should satisfy the following property

$$
\left|\frac{F^{M_{i+1}}(x)-F^{N_{i}}(x)}{M_{i+1}-N_{i}}-\sigma_{i}\right|<\varepsilon_{i} \quad \text { for } x \in K_{i, 1}^{0} .
$$

According to lemma 4.3 this can be achieved.

Step II. Link $X_{i, 1}$ to a sequence which is related to a twist periodic orbit with rotation number $\rho_{i+1}$. If the sequence $X_{i, 2}=\left\{K_{i, 2}^{m}\right\}_{m=0}^{N_{i+1}}$ thus obtained is sufficiently long then there exists according to lemma 4.3 an integer $j_{i+1}$ such that

$$
\begin{array}{ll}
\left|\frac{F^{m}(y)-y}{m}-\rho_{i+1}\right|<\varepsilon_{i+1} & \text { for } y \in K_{i, 2}^{M_{i+1}} \text { and } \\
& j_{i+1} \leq m \leq N_{i+1}-M_{i+1} .
\end{array}
$$

Note that we can choose $N_{i}$ arbitrarily large without altering the construction of $X_{i, 2}$ essentially. Put $J_{i+1}=M_{i+1}+j_{i+1}$. We assume that $N_{i}$ was chosen in such a way that

$$
\begin{array}{ll}
\left|\frac{F^{N_{i}+k}(x)-x}{N_{i}+k}-\rho_{i}\right|<\varepsilon_{i} & \text { for } x \in K_{i, 2}^{0} \text { and } \\
& 0 \leq k \leq J_{i+1}-N_{i} .
\end{array}
$$

From lemma 4.3 and lemma 4.4 we deduce that this condition can be satisfied for $N_{i}$ sufficiently large. Lastly we define $X_{i+1}$ equal to $X_{i, 2}$ and we write $X_{i+1}=\left\{K_{i}^{m}\right\}_{m=0}^{N_{i+1}}$.

For $i \geq 3$ and odd, the construction of $X_{i+1}$ may be sketched as in figure 5.1. 


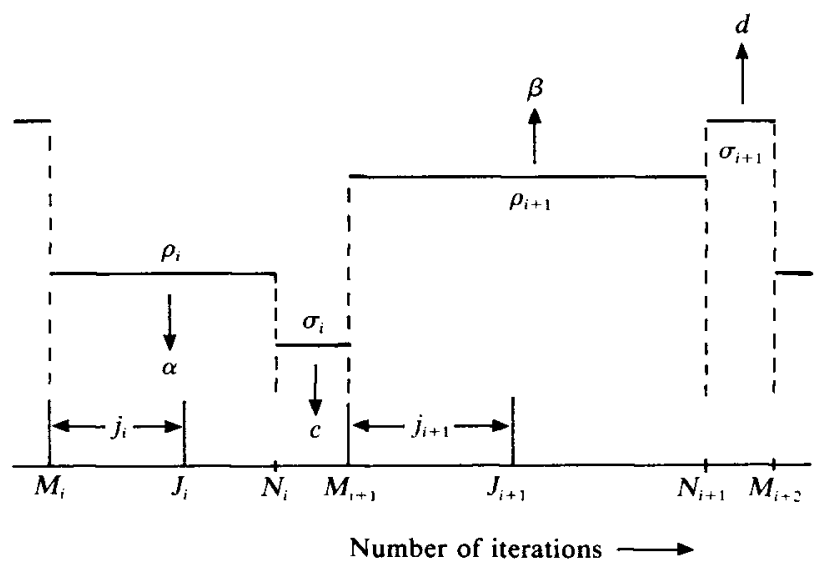

FIGURE 5.1. Sequences which are linked successively in the construction of $X_{i+1}$, where $i \geq 3$ is odd, are displayed by a horizontal. The corresponding rotation number is indicated.

We now finish the proof of the theorem. Since $K_{i}^{0}$ is a non-empty closed set and $K_{i+1}^{0} \subset K_{i}^{0}$ for $i \geq 1$, we have that $K=\bigcap_{i \geq 1} K_{i}^{0} \neq \varnothing$. We claim that $S(z, f)=S\left(z, h_{a, b}\right)$ for $z \in \pi(K)$. Here $h_{a, b} \in \operatorname{End}_{1}^{0}\left(S^{1}\right)$ with $\rho\left(h_{a, b}\right)=[c, d]$ is a circle map induced by an interpolation map $H_{a, b}$. Because of the way we constructed $K_{i}^{0}$ (see also $\S 4$ ) we have $F^{n}\left(K_{i}^{0}\right)=H_{a, b}^{n}\left(K_{i}^{0}\right)$ for $i, n \geq 1$. From this observation the claim follows. Since $z \in \bigcap_{i \geq 1} K_{i}^{0}$ we conclude that $\left(\mathrm{A}_{i}\right)$ holds for all $i \geq 1$ with $x \in \pi^{-1}(z)$. So $[c, d] \subset$ $S(z, f)$ and further $S\left(z, h_{a, b}\right) \subset \rho\left(h_{a, b}\right)=[c, d]$. Hence $z \in K$ satisfies $S(z, f)=[c, d]$.

It remains to prove that $\rho(z, f)=[\alpha, \beta]$. As

$$
\lim _{k \rightarrow \infty} \frac{F^{N_{2 k+1}}(x)-x}{N_{2 k+1}}=\alpha \text { and } \lim _{k \rightarrow \infty} \frac{F^{N_{2 k}}(x)-x}{N_{2 k}}=\beta
$$

for $x \in \pi^{-1}(z)$, it suffices to show that $\alpha \leq\left(F^{m}(x)-x\right) / m \leq \beta$ for $m \geq N_{1}$. Let $m \geq N_{1}$. There exists $i \geq 1$ such that $N_{i} \leq m<N_{i+1}$. The case that $N_{i} \leq m \leq J_{i+1}$ is dealt with by using $\left(C_{i}\right)$. Now let $m=M_{i+1}+k$ with $j_{i+1} \leq k<N_{i+1}-M_{i+1}$, then

$$
\frac{F^{m}(x)-x}{m}=\left[\frac{F^{k}\left(F^{M_{i+1}}(x)\right)-F^{M_{i+1}}(x)}{k}\right] \cdot \frac{k}{m}+\left[\frac{F^{M_{i+1}}(x)-x}{M_{i+1}}\right] \cdot \frac{M_{i+1}}{m},
$$

which is a convex combination of two numbers both belonging to $[\alpha, \beta]$ : by $\left(\mathrm{B}_{i}\right)$ the first one is in an $\varepsilon_{i+1}$-neighbourhood of $\rho_{i+1}$ and the second one belongs to an $\varepsilon_{i}$-neighbourhood of $\rho_{i}$ according to $\left(\mathrm{C}_{i}\right)$. We conclude that $\rho(z, f)=[\alpha, \beta]$. By adapting the proof in an obvious manner one may consider the degenerate cases as settled as well. With this the proof of the theorem is completed.

\section{REFERENCES}

[1] L. Alsedà, J. Llibre, M. Misiurewicz \& C. Simó. Twist periodic orbits and topological entropy for continuous maps of the circle of degree one which have a fixed point. Ergod. Th. \& Dynam. Sys. 5 (1985), 501-517.

[2] L. Alsedà \& J. Llibre. A note on the set of periods for continuous maps of the circle which have degree one. Proc. Amer. Math. Soc. 93 (1985), no. 1, 133-139. 
[3] V. I. Arnol'd. Small denominators I: Mappings of the circumference onto itself. Amer. Math. Soc. Transl., Ser. 2, 46 (1965), 213-284.

[4] R. Bamon, I. P. Malta, M. J. Pacifico \& F. Takens. Rotation intervals of endomorphisms of the circle. Ergod. Th. \& Dynam. Sys. 4 (1984), 493-498.

[5] P. L. Boyland. Bifurcations of circle maps: Arnol'd tongues, bistability and rotation intervals. Commun. Math. Phys. 106 (1986), 353-381.

[6] A. Chenciner, J.-M. Gambaudo \& Ch. Tresser. A remark on degree one endomorphisms of the circle. C.R. Acad. Sc. Paris, t. 299, Sér. I, no. 5 (1984).

[7] R. Ito. Rotation sets are closed. Math. Proc. Camb. Phil. Soc. 89 (1981), 107-111.

[8] M. Misiurewicz. Periodic points of maps of degree one of a circle. Ergod. Th. \& Dynam. Sys. 2 (1982), 221-227.

[9] S. Newhouse, J. Palis \& F. Takens. Bifurcations and stability of families of diffeomorphisms. Publ. Math. I.H.E.S., 57 (1983), 5-71.

[10] H. Poincaré. Euvres complètes, t.1, Gauthier-Villars (1952), 137-158. 